\title{
Phase stability and structural temperature dependence in sodium niobate: A high-resolution powder neutron diffraction study
}

\author{
S. K. Mishra, ${ }^{1}$ R. Mittal, ${ }^{1}$ V. Yu. Pomjakushin, ${ }^{2}$ and S. L. Chaplot ${ }^{1}$ \\ ${ }^{1}$ Solid State Physics Division, Bhabha Atomic Research Centre, Mumbai 400085, India \\ ${ }^{2}$ Laboratory for Neutron Scattering, ETH Zurich and Paul Scherrer Institut, CH-5232 Villigen PSI, Switzerland
}

(Received 19 November 2010; revised manuscript received 9 February 2011; published 7 April 2011)

\begin{abstract}
We report an investigation of structural phase transitions in the technologically important material sodium niobate as a function of temperature on heating over 300-1075 K. Our high-resolution powder neutron diffraction data show a variety of structural phase transitions ranging from nonpolar antiferrodistortive to ferroelectric and antiferroelectric in nature. A discontinuous jump in lattice parameters is found only at about $680 \mathrm{~K}$. This observation along with the reported thermal hysteresis in the dielectric anomaly indicate that the transition of the orthorhombic $P$ to $R$ phase is first order in nature, while other successive phase transitions are of second order. Additional superlattice reflections appear at $680 \mathrm{~K}$ ( $R$ phase) and $770 \mathrm{~K}$ ( $S$ phase) that could be indexed using an intermediate long-period modulated orthorhombic structure whose lattice parameter along the $\langle 001\rangle$ direction is three and six times, respectively, that of the $\mathrm{CaTiO}_{3}$-like $\mathrm{Pbnm}$ structure. The correlation of superlattice reflections with the phonon instability is discussed. The critical exponent $(\beta)$ for the second-order tetragonal to cubic phase transition at about $950 \mathrm{~K}$ corresponds to a value $\beta \approx 1 / 3$, as obtained from the temperature variation of order parameters (tilt angle and intensity of superlattice reflections). It is argued that this exponent is due to a secondorder phase transition close to a tricritical point. Based on our detailed temperature-dependent neutron diffraction studies, a phase diagram of sodium niobate is presented that resolves existing ambiguities in the literature.
\end{abstract}

DOI: 10.1103/PhysRevB.83.134105

PACS number(s): 77.80.-e, 61.05.fm, 77.84.Ek

\section{INTRODUCTION}

The past few decades have seen a tremendous flurry of research interest in perovskite materials due to their fundamental interest and technological applications. ${ }^{1-6}$ In this class of materials, alkali-metal niobates (like potassium sodium niobate and lithium sodium niobate) with ultralarge piezoresponse comparable to $\mathrm{Pb}\left(\mathrm{Zr}_{1-x} \mathrm{Ti}_{x}\right) \mathrm{O}_{3}$ (PZT) have evoked considerable interest as the next-generation ecofriendly leadfree piezoceramics. ${ }^{3-6}$ One of the end members, $\mathrm{NaNbO}_{3}$, is a well-documented antiferroelectric which finds applications in high-density optical storage, enhancing nonlinear optical properties, as hologram recording materials, etc. ${ }^{1,3-6}$ An understanding of its crystallographic structures and phase transitions is of fundamental interest due to its functional properties such as enhanced ferroelectric and piezoelectric response.

$\mathrm{NaNbO}_{3}$ exhibits an unusual complex sequence of temperature-, pressure-, and particle-size-driven structural phase transitions. ${ }^{7-18}$ Above $913 \mathrm{~K}, \mathrm{NaNbO}_{3}$ has a paraelectric cubic phase with space group $P m \overline{3} m$. On cooling, it undergoes a series of antiferrodistortive phase transitions ranging from a paraelectric cubic phase to a ferroelectric rhombohedral phase via intermediate paraelectric tetragonal and orthorhombic phases and an antiferroelectric orthorhombic phase (see Fig. 1 of Ref. 7). These temperature-induced structural phase transitions are driven by the so-called soft-mode mechanism. This concept is built on the assumption that the crystal gets unstable against particular Brillouin zone center and/or boundary phonon vibration modes. ${ }^{19}$ The zone boundary phonon vibrations involve rotations of $\mathrm{NbO}_{6}$ octahedra. The $M_{3}(q=1 / 2,1 / 2,0)$ mode represents in-phase rotation of the adjacent layers (in the same direction $a^{0} a^{0} c^{+}$in Glazer notation), while the $R_{25}(q=1 / 2,1 / 2,1 / 2)$ mode represents the antiphase rotation (opposite manner, $a^{0} a^{0} c^{-}$in Glazer notation). ${ }^{20}$ In soft-mode formalism, cubic to tetragonal
( $T_{2}$ phase) and tetragonal to orthorhombic $\left(T_{1}\right)$ phase transitions are driven by $M_{3}$ and $R_{25}$ phonon modes, respectively. The diffuse x-ray scattering at $R$ and $M$ points into the Brillouin zone is found to be strongly anisotropic and distributed along $\langle 100\rangle$ reciprocal rods which can be reasonably explained by assuming a contribution of the low- frequency zone boundary phonons along the line $T$ (lying between the $M$ and $R$ points). The phase transitions between the orthorhombic $S, R$, and $P$ phases $^{9-11}$ have not been investigated yet, and substantial enlargements of the unit cell and complicated octahedral tilting are not understood.

Further, $\mathrm{NaNbO}_{3}$ exists in a ferroelectric phase at $15 \mathrm{~K}$. The low-temperature transition is driven by zone center and zone boundary $\left(R_{25}\right)$ modes. Our recent low-temperature neutron diffraction studies provided unambiguous experimental evidence for the presence of coexisting ferroelectric $(R 3 c)$ and antiferroelectric phases $(\mathrm{Pbcm})$ over a wide range of temperatures $(15-275 \mathrm{~K})$ in $\mathrm{NaNbO}_{3} .{ }^{7}$ The ferroelectric ordering can be stabilized by application of external field, chemical substitution, and reduction of particle size even at room temperature. ${ }^{6,8}$ The high-temperature phases of ferroelectric and antiferroelectric materials are of primary interest since the dynamics of the dipoles in these phases gives indication about the microscopic nature (e.g., order-disorder versus displacive) of the ferroelectricity itself.

In order to understand the complexity of the structural phase transition sequence in $\mathrm{NaNbO}_{3}$, first-principles calculations were carried out by Vanderbilt and co-workers. ${ }^{14-18}$ The calculations suggest the presence of several competing structural instabilities with similar free energies. The authors included all the distortions involved in the observed structures of $\mathrm{NaNbO}_{3}$ in their model calculations. The calculations predicted the coexistence of zone center and zone boundary phonon instabilities in the cubic phase. However, they could 

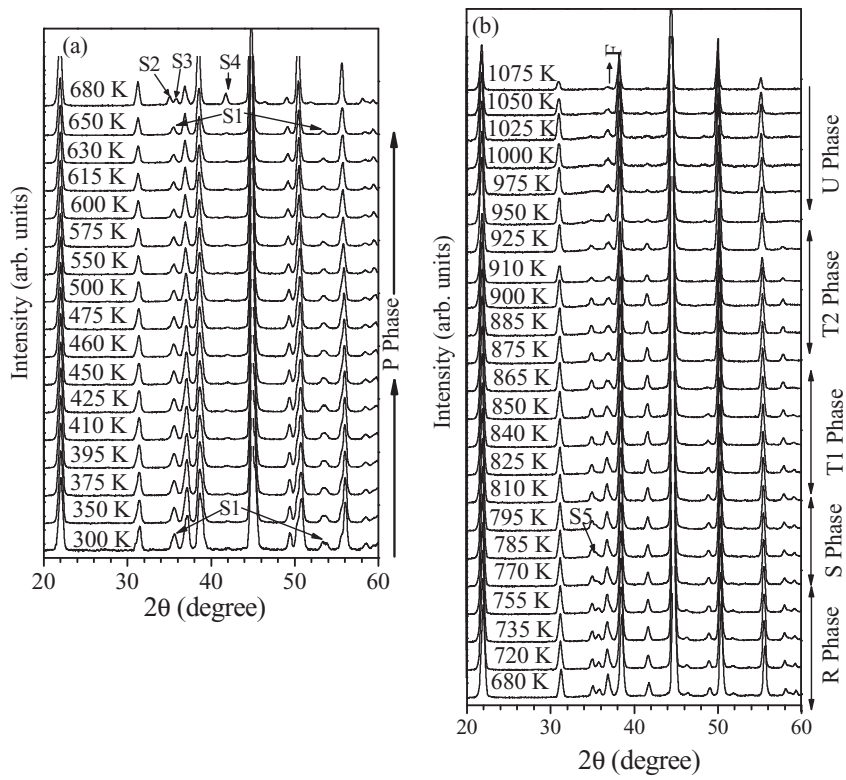

FIG. 1. Evolution of the neutron diffraction patterns for $\mathrm{NaNbO}_{3}$ as a function of temperature. The characteristic superlattice reflections are marked with arrows and labeled as $(S 1, S 2, S 3, S 4$, and $S 5)$. Peak marked with $F$ is due to the furnace materials.

not fully reproduce the observed sequence of structural phase transitions in $\mathrm{NaNbO}_{3}$. The structural and dynamical properties, epitaxial strain, and anomalous LO-TO splitting of $\mathrm{NaNbO}_{3}$ have also been reported.

In spite of the extensive experimental and theoretical studies as a function of temperature, there are numerous controversies surrounding the phase diagram of $\mathrm{NaNbO}_{3}$, especially on the existence of ferroelectric ordering and correctness of the structures of the phases in different temperature regions. Darlington and Knight ${ }^{9}$ have questioned the correctness of the structure at room temperature. They have shown that space group and cell dimensions for the high-temperature $R$ and $S$ phases (stabilized between 633 and $753 \mathrm{~K}$ and 753 and $793 \mathrm{~K}$ ) as reported in the literature ${ }^{9-11}$ are incorrect. Recently, on the basis of work carried out using synchrotron x-ray diffraction, Yuzyuk et al. ${ }^{6}$ proposed a different sequence of structural phase transitions in $\mathrm{NaNbO}_{3}$. However, the correct crystal structures for the two high-temperature $R$ and $S$ phases were still not determined.

The nature of antiferrodistortive phase transition in materials exhibiting perovskite structure has attracted enormous attention in the literature. Mainly, these phase transitions are driven by $R$ point instabilities. We have carried out systematic neutron diffraction measurements, as a function of temperature from 300 to $1075 \mathrm{~K}$, to find the correct crystal structures for high-temperature phases of $\mathrm{NaNbO}_{3}$ and to understand their phase transition behavior. To the best of our knowledge, studies have not been carried out to investigate the nature of antiferrodistortive phase transitions driven by $M$ point instabilities. We have also determined the effective exponent for the second-order cubic to tetragonal phase transition using the intensity of the superlattice reflection and tilt angle. The critical exponent $(\beta)$ is found to be nearly equal to a value $\beta \approx 1 / 3$. It is argued that this exponent is due to a nearly tricritical transition.

\section{EXPERIMENT}

The powder neutron diffraction experiments were carried out at the SINQ spallation source of Paul Scherrer Institute (Switzerland) using the high-resolution diffractometer ${ }^{21}$ for thermal neutrons (HRPT) with wavelength $1.4940 \AA$. The data were collected under high-intensity and high-resolution mode with $\Delta d / d \geqslant 1.8 \times 10^{-3}$. All the data collections were carried out during heating cycles of the sample. The sample was heated up to $1075 \mathrm{~K}$ using a high-temperature tantalum furnace. The structural refinements were performed using the Rietveld refinement program FULLPROF. ${ }^{22}$ In all the refinements, the background was defined by a sixth-order polynomial in $2 \theta$. A Thompson-Cox-Hastings pseudo-Voigt function with axial divergence asymmetry function was chosen to define the profile shape for the neutron diffraction peaks. Except for the occupancies of the atoms, all other parameters, i.e., scale factor, zero correction, background, and half-width parameters along with mixing parameters, lattice parameters, positional coordinates, and thermal parameters, were refined. All the refinements have used the data over the full angular range of $4^{\circ} \leqslant 2 \theta \leqslant 164^{\circ}$; although in various figures only a limited range is shown for clarity.

\section{RESULTS AND DISCUSSION}

\section{A. Evolution of neutron diffraction as a function of temperature}

Figure 1 depicts a portion of the powder neutron diffraction patterns of $\mathrm{NaNbO}_{3}$ as a function of temperature in the range from 300 to $1075 \mathrm{~K}$ during the heating cycle. We shall discuss the structures starting from the highest-symmetry cubic phase that occurs at the highest temperature followed by the other phases occurring at lower temperatures. At the highest temperature $(T=1075 \mathrm{~K})$, all the Bragg reflections present in powder diffraction patterns could be indexed as main cubic perovskite reflections. The reflection marked with an arrow and labeled as $F\left(2 \theta=35.1^{\circ}\right)$ in the diffraction pattern of cubic phase is from the furnace material [see Fig. 1(b)]. Below 950 and $900 \mathrm{~K}$, two different superlattice reflections appear at $2 \theta=35.0^{\circ}$ and $37.0^{\circ}$ (near to $F$ ), respectively. These superlattice reflections are associated with zone boundary $M$ and $R$ point instabilities, respectively. Further, below $810 \mathrm{~K}$, an additional set of superlattice reflections appears centered at $2 \theta=35.4^{\circ}$ [marked with an arrow and label as $S 5$ in Fig. 1(b)], and some of them diminish followed by enhancement of the intensity of the superlattice reflection at $2 \theta=35.8^{\circ}$ [marked with arrows and labeled as $S 3$ in Fig. 1(a)], which disappears below $680 \mathrm{~K}$. Below $680 \mathrm{~K}$, the superlattice reflections present at $2 \theta=35.0^{\circ}, 35.8^{\circ}$, and $41.7^{\circ}$ [marked with arrows and labeled as $S 2, S 3$, and $S 4$ in Fig. 1(a)] vanish and different superlattice reflections appear around $2 \theta=35.6^{\circ}$ and $53.5^{\circ}$ [marked with an arrow and labeled as $S 1$ in Fig. 1(a)]. The intensity of these reflections increases gradually with decrease of temperature and reflections are present at room temperature.

$\mathrm{NaNbO}_{3}$ has an antiferroelectric phase $(\mathrm{Pbcm})$ at room temperature. In general, an antiferroelectric phase consists of two or more sublattice polarizations of antiparallel nature, 
TABLE I. Structural parameters of $\mathrm{NaNbO}_{3}$ obtained by neutron diffraction at $1075 \mathrm{~K}$. The cubic lattice parameter is $3.9507(2) \AA$ in the space group $P m \overline{3} m$. The unwritten thermal parameters $U_{i j}$ are zero.

\begin{tabular}{|c|c|c|c|c|c|c|}
\hline \multicolumn{7}{|c|}{ Temperature $=1075 \mathrm{~K}(U$ phase $)$} \\
\hline \multirow[b]{2}{*}{ Atoms } & \multicolumn{3}{|c|}{ Positional coordinates } & \multicolumn{3}{|c|}{ Thermal parameter $\left(10^{-2} \AA^{2}\right)$} \\
\hline & $X$ & $Y$ & $Z$ & $U 11$ & $U 22$ & $U 33$ \\
\hline $\mathrm{Na}$ & 0 & 0 & 0 & $7.32(9)$ & $7.32(9)$ & $7.32(9)$ \\
\hline $\mathrm{Nb}$ & 0.5 & 0.5 & 0.5 & $1.96(4)$ & $1.96(4)$ & $1.96(4)$ \\
\hline $\mathrm{O}$ & 0.5 & 0.5 & 0 & $7.40(8)$ & $7.40(8)$ & $1.94(6)$ \\
\hline \multicolumn{7}{|c|}{$R_{B}=1.79 ; R_{p}=6.89 ; R_{w p}=8.45 ; R_{\text {expt }}=6.82 ; \chi^{2}=1.54$} \\
\hline
\end{tabular}

which in turn give rise to superlattice reflections in the diffraction pattern. Superlattice reflections around $2 \theta=35.6^{\circ}$ and $53.5^{\circ}$ are some of the strongest antiferroelectric peaks and are marked with arrows and labeled as $S 1$ in Fig. 1(a). Thus, the disappearance and reappearance of superlattice reflections in powder neutron diffraction provide unambiguous evidence for structural phase transitions with temperature in sodium niobate. Superlattice reflections, in different temperature regimes, are also present at higher angles with prominent intensities. The details of symmetry and structure in different temperature regime are discussed in the next section.

\section{B. Rietveld analysis of powder neutron diffraction data \\ 1. Cubic (U) phase (above $T>950 \mathrm{~K}$ )}

The powder neutron diffraction patterns above $950 \mathrm{~K}$ are analyzed on the basis of an ideal cubic perovskite structure with the space group $P m \overline{3} m$. The detailed structural parameters and goodness of fit for the cubic phase of sodium niobate at $1075 \mathrm{~K}$, as obtained from neutron diffraction data, are given in
Table I. The fit between the observed and calculated profiles is satisfactory [Fig. 2(a)]. Refinement with anistropic atomic displacement parameters gives a better fit. Strongly anisotropic thermal vibrations of oxygen atoms, with the site symmetry $4 / \mathrm{mmm}$, are observed. In the cubic phase, as seen in Table I, the oxygen atom at $(0.5,0.50)$ has $U_{11}=U_{22} \gg U_{33}, U_{i j}=0$, and $U_{33} \approx U(N b)$. This signifies large-amplitude libration motion of the $\mathrm{NbO}_{6}$ octahedra, and suggests structural phase transitions driven by octahedral librational $M_{3}$ and $R_{25}$ modes at lower temperatures. ${ }^{23}$

\section{Tetragonal $\left(T_{1}\right)$ and orthorhombic $\left(T_{2}\right)$ phases (temperature range $825<T<950 \mathrm{~K}$ )}

The presence of superlattice reflections at $2 \theta=35.0^{\circ}$ and $37.0^{\circ}$ below 950 and $865 \mathrm{~K}$, respectively, in powder neutron diffraction patterns indicates the structural phase transitions from the cubic phase. These superlattice reflections arise due to condensation of $M$ and $R$ zone boundary phonons and characteristics of the tetragonal and orthorhombic phases, respectively. Thus, for the temperature ranges $865<T$
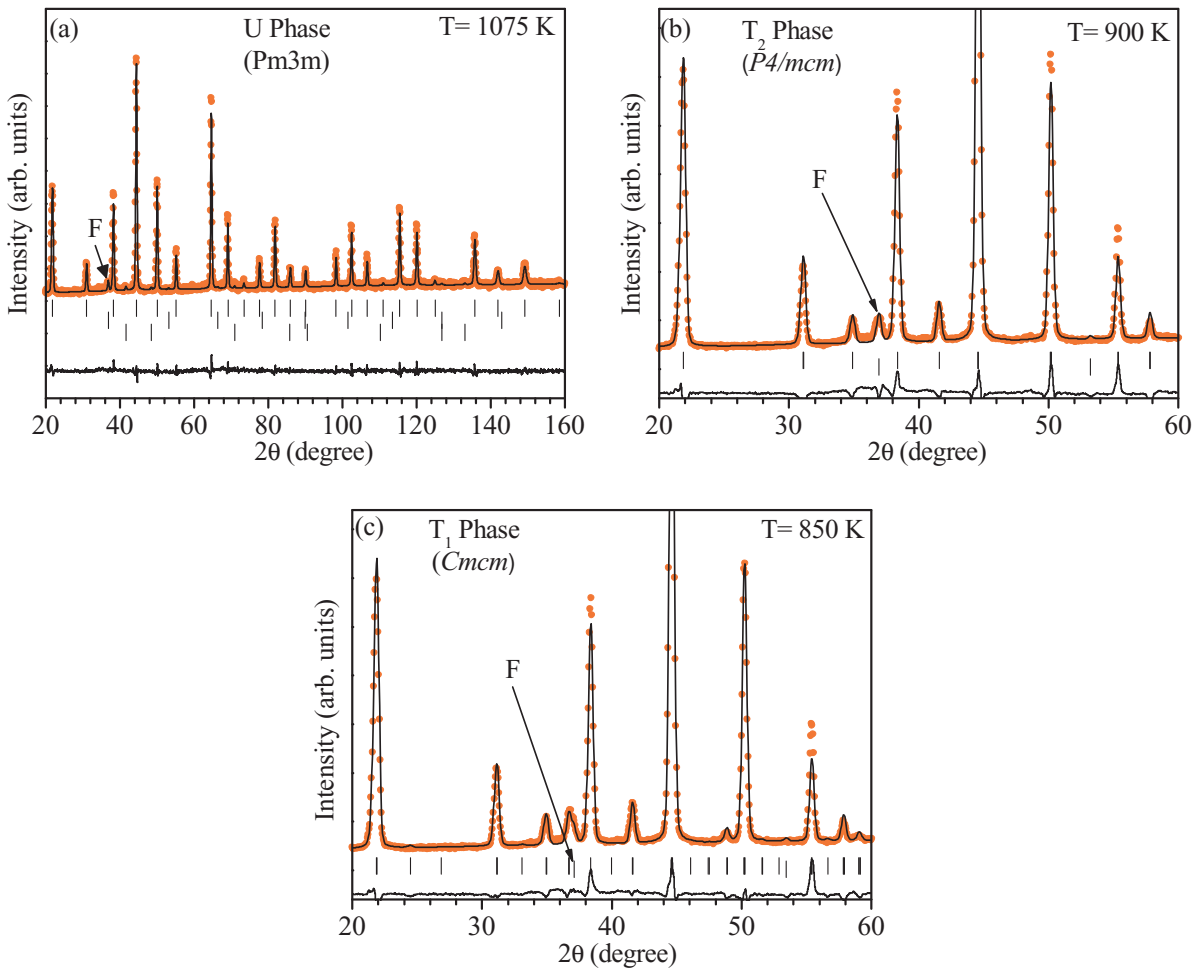

FIG. 2. (Color online) Observed (open circles), calculated (continuous line), and difference (bottom line) profiles obtained after the Rietveld refinement of $\mathrm{NaNbO}_{3}$ using (a) cubic $(P m \overline{3} m)$, (b) tetragonal $(P 4 / m b m)$, and (c) orthorhombic $(\mathrm{Cmcm})$ phases. Peak marked with $F$ is due to the furnace materials. In the cubic phase, middle and lower vertical tick marks above the difference profiles correspond to the furnace and thermocouple materials, respectively. The $R$ factors obtained by Rietveld refinement of neutron diffraction data are $R_{B}=4.79 ; R_{p}=4.74$; $R_{w p}=6.56 ; \quad R_{\text {expt }}=2.89 ; \quad \chi^{2}=5.15$ for tetragonal $(P 4 / \mathrm{mbm})$ and $R_{B}=$ 4.93; $R_{p}=4.24 ; R_{w p}=6.72 ; R_{\text {expt }}=$ 2.93; $\chi^{2}=5.26$ orthorhombic $(\mathrm{Cmcm})$ phases. 
TABLE II. Structural parameters of $\mathrm{NaNbO}_{3}$ obtained by Rietveld refinement of neutron diffraction at $770(S$ phase $)$ and $680 \mathrm{~K}(R$ phase $)$ using Pbnm space group.

\begin{tabular}{|c|c|c|c|c|c|c|c|c|c|}
\hline \multicolumn{5}{|c|}{ (a) Temperature $=770 \mathrm{~K}(S$ phase $)$} & \multicolumn{5}{|c|}{ (b) Temperature $=680 \mathrm{~K}$ ( $R$ phase $)$} \\
\hline \multirow[b]{2}{*}{ Atoms } & \multicolumn{3}{|c|}{ Positional coordinates } & \multirow{2}{*}{$\begin{array}{c}\text { Thermal parameter } \\
\qquad B(\AA)^{2}\end{array}$} & \multirow[b]{2}{*}{ Atoms } & \multicolumn{3}{|c|}{ Positional coordinates } & \multirow{2}{*}{$\begin{array}{c}\text { Thermal parameter } \\
\qquad B(\AA)^{2}\end{array}$} \\
\hline & $X$ & $Y$ & $Z$ & & & $X$ & $Y$ & $Z$ & \\
\hline $\mathrm{Na} 1$ & $0.5260(2)$ & $-0.0073(7)$ & $0.0410(7)$ & $0.710(1)$ & Na1 & $0.5138(1)$ & $0.4984(1)$ & 0.2500 & $2.185(3)$ \\
\hline $\mathrm{Na} 2$ & $0.5299(9)$ & $-0.0315(7)$ & $0.1182(9)$ & $2.026(6)$ & $\mathrm{Na} 2$ & $0.9863(2)$ & $0.0109(3)$ & $0.0872(1)$ & $2.990(1)$ \\
\hline $\mathrm{Na} 3$ & $0.5107(5)$ & $-0.0146(5)$ & $0.2092(2)$ & $3.132(6)$ & $\mathrm{Nb} 1$ & 0.5000 & 0.0000 & 0.0000 & $1.013(1)$ \\
\hline $\mathrm{Nb} 1$ & 0.0000 & 0.0000 & 0.0000 & $0.844(4)$ & $\mathrm{Nb} 2$ & $0.4981(1)$ & $0.0041(2)$ & $0.3323(2)$ & $0.868(1)$ \\
\hline $\mathrm{Nb} 2$ & $0.0068(8)$ & $0.0065(2)$ & $0.0822(2)$ & $0.878(3)$ & $\mathrm{O} 1$ & $0.5315(1)$ & $-0.0267(1)$ & 0.2500 & $1.512(2)$ \\
\hline $\mathrm{Nb} 3$ & $-0.0100(5)$ & $-0.0005(1)$ & $0.1680(6)$ & $1.022(4)$ & $\mathrm{O} 2$ & $0.0597(2)$ & $0.4824(2)$ & $0.0835(1)$ & $2.684(4)$ \\
\hline $\mathrm{Nb} 4$ & $0.0017(3)$ & $-0.0012(4)$ & 0.25000 & $1.082(3)$ & $\mathrm{O} 3$ & $0.7553(1)$ & $0.2442(3)$ & $0.0040(2)$ & $2.210(5)$ \\
\hline $\mathrm{O} 1$ & $0.0520(4)$ & $-0.0007(7)$ & $0.0415(1)$ & $3.225(3)$ & $\mathrm{O} 4$ & $0.7070(3)$ & $0.2932(1)$ & $0.3391(4)$ & $0.453(3)$ \\
\hline $\mathrm{O} 2$ & $0.0368(4)$ & $0.0557(6)$ & $0.1243(1)$ & $2.636(4)$ & O5 & $0.7151(2)$ & $0.2885(3)$ & $0.6734(3)$ & $0.573(4)$ \\
\hline $\mathrm{O} 3$ & $-0.0207(5)$ & $0.0005(4)$ & $0.2083(3)$ & $0.773(3)$ & & & & & \\
\hline $\mathrm{O} 4$ & $0.1990(7)$ & $0.2903(6)$ & $0.0028(6)$ & $0.001(1)$ & & & & & \\
\hline O5 & $0.2663(2)$ & $0.2358(1)$ & $0.0817(8)$ & $1.811(2)$ & & & & & \\
\hline O6 & $0.7290(1)$ & $0.7636(3)$ & $0.0827(1)$ & $1.977(2)$ & & & & & \\
\hline $\mathrm{O} 7$ & $0.2028(1)$ & $0.2851(1)$ & $0.1692(4)$ & $0.541(1)$ & & & & & \\
\hline O8 & $0.7992(2)$ & $0.6980(5)$ & $0.1692(3)$ & $1.062(2)$ & & & & & \\
\hline O9 & $0.2055(3)$ & $0.2737(5)$ & 0.2500 & $2.482(3)$ & & & & & \\
\hline O10 & $0.7792(5)$ & $0.7163(1)$ & 0.2500 & $0.668(3)$ & & & & & \\
\hline \multicolumn{5}{|c|}{$\begin{array}{l}\text { Lattice parameters }(\AA) \\
A=5.5555(2)(\AA) ; B=5.5556(2)(\AA) \\
C=47.1489(9) ; \text { volume }=1455.230(8)(\AA)^{3} \\
R_{B}=5.84 ; R_{p}=10.2 ; R_{w p}=10.4 ; R_{\text {expt }}=4.01 ; \\
\chi^{2}=6.77\end{array}$} & \multicolumn{5}{|c|}{$\begin{array}{l}\text { Lattice parameters }(\AA) \\
A=5.5459(1)(\AA) ; B=5.5505(1)(\AA) \\
C=23.5229(3) ; \text { volume }=723.087(5)(\AA)^{3} \\
R_{B}=6.85 ; R_{p}=10.3 ; R_{w p}=10.35 ; R_{\exp }=4.05 \\
\chi^{2}=6.62\end{array}$} \\
\hline
\end{tabular}

$<950 \mathrm{~K}$ and $810<\mathrm{T}<865 \mathrm{~K}$ we refined the powder neutron diffraction data using tetragonal symmetry (space group $P 4 / \mathrm{mbm}$ ) and orthorhombic symmetry (space group $\mathrm{Cmcm}$ ), respectively. The results of Rietveld refinements are shown in Figs. 2(b) and 2(c). We note that the contamination due to the furnace material decreased in the lower temperature range.

\section{Orthorhombic S phases (temperature range $755<T<825 \mathrm{~K}$ )}

The main feature of this phase is the appearance of additional superlattice reflections beyond those in the $T_{2}$ phase at $2 \theta=35.4^{\circ}$ and $35.8^{\circ}$. These superlattice reflections, marked with arrows and label as $S_{5}$ in Fig. 1(b), could not be indexed using the symmetry and space group of the orthorhombic $\left(T_{2}\right)$ phase. The absence of splitting in the main perovskite reflections and the presence of different superlattice reflections suggest that the multiplicity of the cell is increased with respect to the orthorhombic $T_{2}$ phase. The x-ray diffraction measurements by Ahtee et al. ${ }^{10}$ showed that the $S$ phase has orthorhombic symmetry with Pnmm space group and cell dimension $2 \times 2 \times 2$ with respect to the elementary perovskite cubic cell. However, Rietveld refinement of neutron diffraction data using the model proposed by Ahtee et al. ${ }^{10}$ did not account for the superlattice reflection at $2 \theta=35.4^{\circ}$.

In order to index the additional superlattice reflection, we explored various possibilities and found that further multiplication of the lattice parameter along [001] of the orthorhombic $R$ phase (see next section) indexed all the reflections. Thus the
$S$ phase has orthorhombic structure with space group Pbnm and cell dimensions $\sqrt{2} \times \sqrt{2} \times 12$ with respect to the elementary perovskite cell. This unit cell indicates that the structure of the $S$ phase of $\mathrm{NaNbO}_{3}$ is a modulated structure in which the lattice parameter along [001] is twice the $C_{0}$ lattice parameter of the $R$ phase.

In the space group Pbnm, for the description of the crystal structure, we used the following axes: $\mathbf{A}_{0}=-\mathbf{a}_{p}+\mathbf{b}_{p}, \mathbf{B}_{0}=$ $\mathbf{a}_{p}+\mathbf{b}_{p}$, and $\mathbf{C}_{0}=12 \mathbf{c}_{p}$, where $a_{p}, b_{p}$, and $c_{p}$ correspond to the parent cubic phase perovskite. The orthorhombic unit cell contains 24 formula units. The detailed structural parameters and goodness of fit for the $S$ phase of sodium niobate at $770 \mathrm{~K}$, as obtained from neutron diffraction data, are given in Table II(a). The fit between the observed and calculated profiles is quite satisfactory and includes the weak superlattice reflections as shown in Fig. 3(a). These superlattice reflections were also seen by Darlington and Knight ${ }^{9}$ using high-resolution neutron powder diffraction data. To show the accountabilities of these superlattice reflections we have further plotted neutron data in term of $d$ spacing (A) [see Fig. 3(b)].

\section{Analysis of $R$ phase (temperature range $650<T<770 \mathrm{~K}$ )}

In the $R$ phase, the characteristic superlattice reflections appear at $2 \theta=35.0^{\circ}, 35.8^{\circ}, 37^{\circ}$, and $41.7^{\circ}$, etc. We note that the superlattice reflection in the $S$ phase at $35.4^{\circ}$ does not occur in the $R$ phase. Moreover, the intensity of the reflection at $35.8^{\circ}$ is enhanced in the $R$ phase compared to that in the 

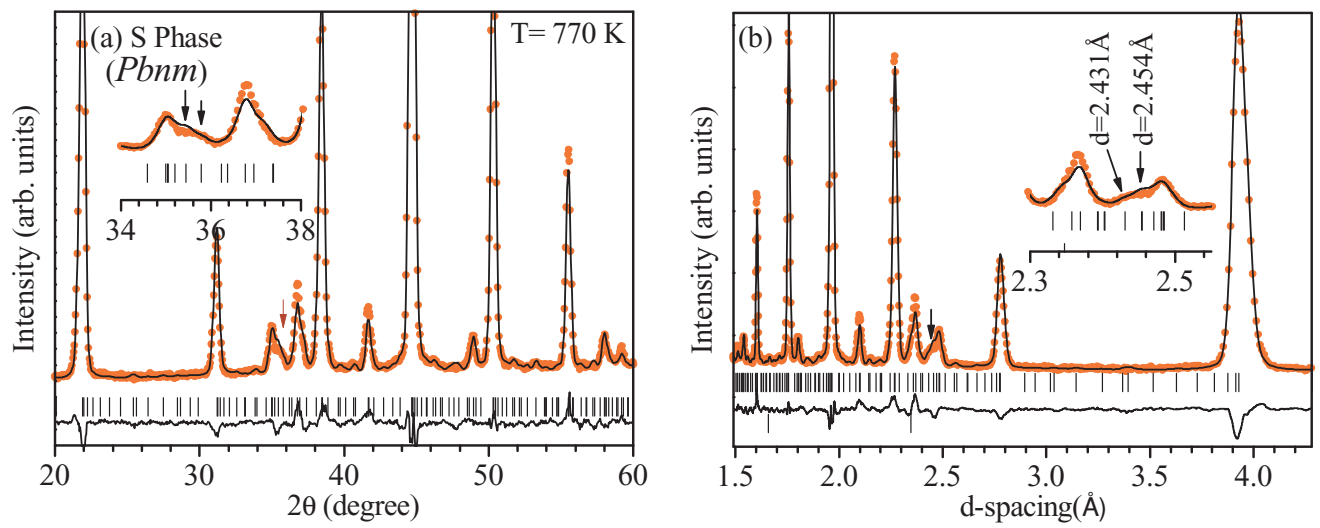

FIG. 3. (Color online) Observed (open circles), calculated (continuous line), and difference (bottom line) profiles obtained after the Rietveld refinement of $\mathrm{NaNbO}_{3}$ using long-period modulated orthorhombic structure with space group Pbnm at $770 \mathrm{~K}$. (b) In order to have an easy comparison with the Darlington and Knight work, ${ }^{9}$ the data have been plotted again in term of $d$ spacing $(\AA)$. Inset shows the accountabilities of the characteristic superlattice reflections appearing in the $S$ phase at $2 \theta=35.4^{\circ}$ and $35.8^{\circ}$ (in $d$-spacing terms $d=2.454$ and $2.431 \AA$ ).

$S$ phase. The superlattice reflections in the $R$ phase have $(h, k / 6, l) ;(h / 2, k / 2, l)$, and $(h / 2, k / 2, l / 2)$ indices (in terms of the elementary perovskite cell). The reflections appear due to condensation of $D T(q=0,1 / 6,0), M(q=1 / 2,1 / 2,0)$, and $R(q=1 / 2,1 / 2,1 / 2)$ soft modes. The structure of the $R$ phase is not yet well investigated using neutron diffraction data. Sakowski has studied the structure of the $R$ phase; however, the structural parameters were not refined. ${ }^{9}$ The author has shown that the $R$ phase has orthorhombic structure with space group Pnmm with cell dimension $2 \times 6 \times 2$ times as large as that of the elementary perovskite cell.

The Miller indices of the superlattice reflections based on an elementary perovskite cell give information about the nature of the octahedral tilts in the structure. Superlattice reflections with all-odd integered indices ("odd-odd-odd" i.e., "000" type in Gazer notation) and two-odd and one-even integered indices result from antiphase $(-$ tilt $)$ and in-phase $(+$ tilt $)$ tilting of the adjacent oxygen octahedron. This is due to structural phase transitions driven by softening and freezing of the phonons at $R(q=1 / 2,1 / 2,1 / 2)$ and $M(q=1 / 2,1 / 2,0)$ points of the cubic Brillouin zone, respectively. The presence of superlattice $(h, k / 6, l),(h / 2, k / 2, l)$, and $(h / 2, k / 2, l / 2)$ type reflections in powder neutron diffraction pattern confirms that these phase transition involve $D T, M$, and $R$ soft modes.

To find the most probable space group we coupled $D T, M_{3}^{+}$, and $R_{4}^{+}$irreducible representations (irreps) using the software package ISOTROPY $2000^{24}$ and obtained many space groups. The $P$ phase of $\mathrm{NaNbO}_{3}$ is orthorhombic $(P b c m)$ and undergoes a first-order phase transition to the $R$ phase. Thus we imposed the constraints that the $R$ phase has higher symmetry than the $P$ phase $(P b c m)$, is primitive in nature and a subgroup of $\mathrm{Cmcm}$, and does not follow a group-subgroup relation for $\mathrm{Pbcm}$. This constraint reduced the number of possible space groups for the $R$ phase. We find that the orthorhombic structure with space group Pnma (different setting of Pbnm) with cell dimensions $\sqrt{2} \times 6 \times \sqrt{2}$ with respect to the elementary perovskite cell accounts for all the reflection in the $R$ phase (see Fig. 4). In the space group Pbnm, for the description of the crystal structure, we used the following axes: $\mathbf{A}_{0}=-\mathbf{a}_{p}+\mathbf{b}_{p} \mathbf{B}_{0}=\mathbf{a}_{p}+\mathbf{b}_{p}$, and $\mathbf{C}_{0}=6 \mathbf{c}_{p}$, where $a_{p}, b_{p}$, and $c_{p}$ are elementary perovskite cell parameters. The orthorhombic unit cell contains 12 formula units. The detailed structural parameters and goodness of fit for the $R$ phase of sodium niobate at $680 \mathrm{~K}$, as obtained from neutron diffraction data, are given in Table II(b). The
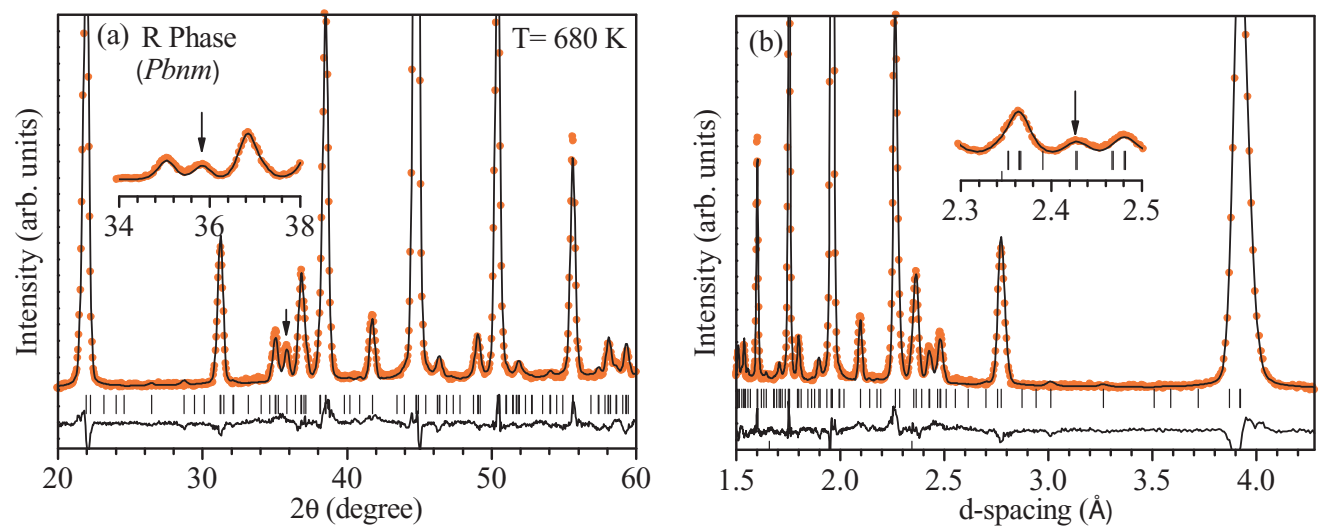

FIG. 4. (Color online) Observed (open circles), calculated (continuous line), and difference (bottom line) profiles obtained after the Rietveld refinement of $\mathrm{NaNbO}_{3}$ using orthorhombic Pbnm space groups at $680 \mathrm{~K}$. The arrow shows the accountability of the additonal superlattice reflection. (b) In order to have an easy comparison with the Darlington and Knight work, ${ }^{9}$ the data have been plotted again in terms of $d$ spacing $(\AA)$. Inset shows the accountabilities of the characteristic superlattice reflections appearing in the $R$ phase. 

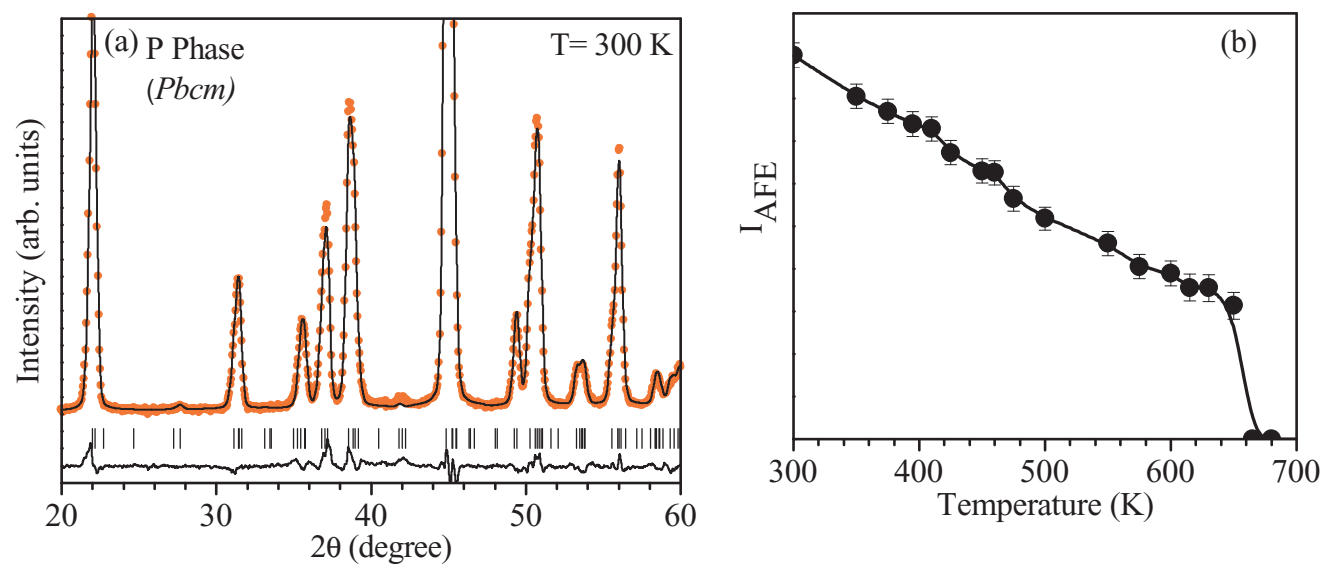

FIG. 5. (Color online) Observed (open circles), calculated (continuous line), and difference (bottom line) profiles obtained after the Rietveld refinement of $\mathrm{NaNbO}_{3}$ using orthorhombic $\mathrm{Pbcm}$ space groups at $300 \mathrm{~K}$. (b) Variation of the integrated intensity of one of the strongest antiferroelectric reflections appearing around $2 \theta=35.6^{\circ}$ with temperature. The $R$ factors obtained by Rietveld refinement of neutron diffraction data for the orthorhombic antiferroelectric phase are $R_{B}=4.24 ; R_{p}=4.24 ; R_{w p}=5.33 ; R_{\text {expt }}=3.67 ; \chi^{2}=2.11$.

fit between the observed and calculated profiles is quite satisfactory and includes the weak superlattice reflections. For comparison, we have also carried out Rietveld refinement using the Pnmm space group with cell dimension $2 \times 6 \times 2$. We found higher $\chi^{2}$ with the Pnmm space group compared to the orthorhombic Pbnm space group although the former involved more parameters in the refinement.

\section{Analysis of P phase (temperature range $300<T<650 \mathrm{~K}$ )}

The powder neutron diffraction data of the $P$ phase $(300-$ $650 \mathrm{~K})$ include superlattice reflections which assume the indices $(h, k, l / p)$, where $h, k$, and $l$ are integers, and $p$ (four in this case) gives the multiple along the [001] direction with respect to the equivalent cubic perovskite cell. These superlattice reflections are characteristic of the antiferroelectric phase. The powder neutron data have been analyzed using the orthorhombic structure with space group $\mathrm{Pbcm}$ (different setting of Pbma). The size of the orthorhombic unit cell is $\sqrt{2} \times \sqrt{2} \times 4$ times as large as that of the high-temperature cubic cell (elementary perovskite cell). Figure 5(a) depicts the observed, calculated, and difference profiles obtained by Rietveld analysis of the powder neutron data at $300 \mathrm{~K}$. The fit between the observed and calculated profiles is quite good and includes all the weak superlattice reflections. This confirms the orthorhombic structure with space group $\mathrm{Pbcm}$ of $\mathrm{NaNbO}_{3}$ in the temperature range from 300 to $650 \mathrm{~K}$ (Fig. 1). Based on synchrotron x-ray diffraction diffraction studies, Yuzyuk et $a l .{ }^{6}$ proposed that the $P$ phase (from 250 to $650 \mathrm{~K})$ consists of three phases: monoclinic $(P m)$ between 250 and $410 \mathrm{~K}$, incommensurate between 410 and $460 \mathrm{~K}$, and orthorhombic $(\mathrm{Po})$ between 460 and $633 \mathrm{~K}$. In view of this, we have collected data in a small temperature interval and carried out careful Rietveld analysis. We have determined the structure using neutron diffraction, which offers advantages over x-ray diffraction, especially in the accurate determination of oxygen positions that are found to be responsible for subtle changes in structures. However, we did not find any signature for the presence of the above phase during Rietveld analysis of powder neutron data. The intensity of the characteristic antiferroelectric reflection is found to decrease with increasing temperature and finally vanish at $680 \mathrm{~K}$ [see Fig. 5(b) ].

\section{Variation of lattice parameters}

Variation of lattice parameters with temperature obtained from the Rietveld refinements is plotted in Fig. 6. The [110], $[1, \overline{1}, 0]$, and [001] directions of the cubic phase correspond to the [100], [010], and [001] directions, respectively, in all the orthorhombic $P$ (space group $P b c m$ ), $R$ and $S$ (space group $P b n m$ ) phases, and tetragonal (space group $P 4 / \mathrm{mbm}$ ) phases. On the other hand, the lattice vectors of the orthorhombic $T_{1}$ phase (space group $\mathrm{Cmcm}$ ) coincide with those of the original $\langle 100\rangle$ cubic phase vectors. For the sake of easy comparison with the corresponding cell parameters of the various phases of sodium niobate, we have plotted equivalent elementary lattice parameters instead of lattice parameters corresponding to the different phases in Fig. 6. It is evident from the figure that in the antiferroelectric orthorhombic phase ( $P$ phase), the $a_{p}$ and $c_{p}$ parameters increase while the $b_{p}$ parameter decreases with increase in temperature. We observe a discontinuous jump of lattice parameters at about $680 \mathrm{~K}$. Shirane et al. ${ }^{25}$ also observed thermal hysteresis in the dielectric anomaly around

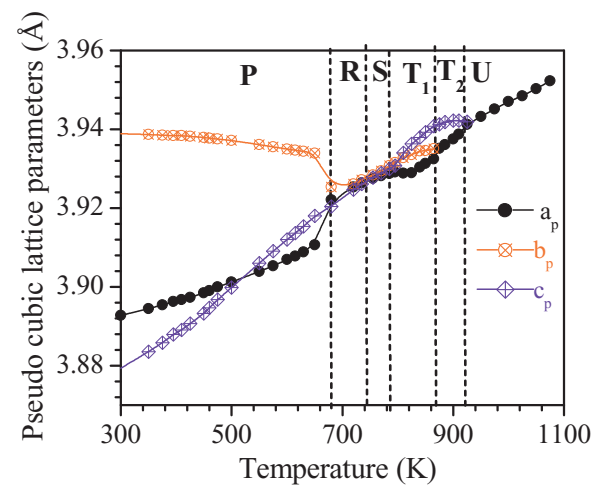

FIG. 6. (Color online) The variation of the pseudocubic lattice parameters as a function of temperature obtained after Rietveld refinement for $\mathrm{NaNbO}_{3}$. 
this temperature. These two observations suggest a first-order phase transition.

Around $680 \mathrm{~K}$, all the lattice parameters come close to each other and increase continuously with increase of temperature. The absence of abrupt change in the lattice parameters at the $R$ to $S$ phase transition temperature suggests that the phase transition is of second order in nature. As we further increase the temperature, the $a_{p}, b_{p}$, and $c_{p}$ lattice parameters of the orthorhombic ( $\mathrm{Cmcm}$ phase) phase increase and the $a_{p}$ parameter approaches $b_{p}$ around $865 \mathrm{~K}$. At this temperature, the sample undergoes an orthorhombic to tetragonal phase transition. Further, as temperature increases, the $c_{p}$ lattice parameter decreases and finally approaches the $a_{p}$ parameter, and the sample transforms into the cubic phase. The continuous change in the lattice parameters reveals that the successive phase transformations from orthorhombic $R$ to high-temperature cubic phase are not of a strong first order in nature.

\section{PHASE TRANSITIONS}

Distinct changes or vanishing of the intensity of superlattice reflections observed in the temperature dependence of neutron diffraction patterns of $\mathrm{NaNbO}_{3}$ clearly reveal that it undergoes a series of phase transitions as a function of temperature, and consistent with previous observations from the literature. ${ }^{9-11}$ Based on powder synchrotron x-ray diffraction studies, Yuzyuk et al. ${ }^{6}$ claimed existence of three phases over the temperature range 250 to $633 \mathrm{~K}$, namely, monoclinic $(P m)$ between 250 and $410 \mathrm{~K}$, incommensurate between 410 and $460 \mathrm{~K}$, and orthorhombic (Po) between 460 and $633 \mathrm{~K}$. We are able to refine all the diffraction patterns over 300 to $633 \mathrm{~K}$ with the orthorhombic phase and do not find any evidence of a phase transition over the temperature range. However, it may be noted from Fig. 7 that around $500 \mathrm{~K}$, the

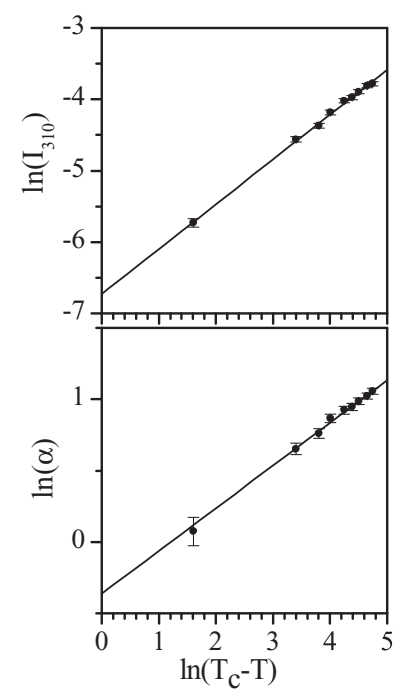

FIG. 7. Logarithmic plots of the variation of the integrated intensity (I) (in arbitrary units) of (310) reflection and tilt angle $(\alpha)$ (in degrees) with temperature. The symbols represent experimental values while the continuous line corresponds to the least-squares fit in the temperature range $0.89 T_{c} \leqslant T<0.99 T_{c}$. The transition temperature $\left(T_{c}\right)$ is found to be $953 \mathrm{~K}$. plots of the lattice parameters $a_{p}$ and $c_{p}$ cross each other. The microscopic origins of various phases that stabilized over 10 to $1075 \mathrm{~K}$ are discussed below.

Recently, Izumi et al. ${ }^{12}$ carried out a detailed inelastic neutron scattering study in the cubic phase of $\mathrm{NaNbO}_{3}$. The measurements show gradual softening of transverse acoustic (TA) phonon modes at the zone boundary $M(1 / 2,1 / 2,0)$ and $R(1 / 2,1 / 2,1 / 2)$ points, indicating the instabilities of the in-phase and out-of-phase rotations of the oxygen octahedron about the [001] direction. The softening of these modes suggests a low-lying flat transverse acoustic phonon-dispersion relation along the zone boundary $M-R$ line ( $T$ line). The sequence of phase evolution depends on the condensation sequence of the soft modes. The $R_{25}$ mode is threefold degenerate and the $M_{3}$ mode is nondegenerate. The triply degenerate $R_{25}$ soft mode could be treated as made up of three components corresponding to the rotations of the octahedra around three separate [001] axes. If only one component condensed at the transition point, the resulting structure would be tetragonal $I 4 / \mathrm{mcm}$. On the other hand, coupled condensation of all the three components would give a rhombohedral $R 3 c$ structure. Similarly, depending on the number of condensed $M_{3}$ phonons in the $\mathbf{q}$ star (successively one, two, and three), the symmetry-related second-order phase transition from the cubic perovskite could be to $P m \overline{3} m$, $P 4 / \mathrm{mbm}, I 4 / \mathrm{mmm}$, and $I m 3$, respectively.

In the case of $\mathrm{NaNbO}_{3}$, in contrast to other perovskites like $\mathrm{SrTiO}_{3}, \mathrm{CaTiO}_{3}, \mathrm{KNbO}_{3}$, etc., the $M_{3}$ phonon condenses first on cooling, followed by the $R_{25}$ phonon on further cooling. The first structural transformation is from cubic $P m \overline{3} m$ to tetragonal $\left(T_{1}\right) P 4 / m b m$ structure, doubling the unit cell in the plane perpendicular to the rotation axes though one $M_{3}$ mode condensation. Further, condensation of the $R_{25}$ phonon leads to the orthorhombic $\mathrm{Cmcm}\left(T_{2}\right)$ phase. This is an example of the $M_{3}$ mode instability in the cubic to tetragonal phase transition in a crystal of perovskite structure. Further, the observation of (1) a low-lying flat transverse acoustic phonon dispersion relation along the zone boundary $M-R$ line and (2) strongly anisotropic diffuse scattering ${ }^{11}$ that is distributed along $\langle 100\rangle$ reciprocal rods at $R$ and $M$ point strongly indicate that the low-frequency zone boundary phonons lying between the $M$ and $R$ points also contribute to the transitions. These phonons play an important role in stabilization of different $\left(P, S\right.$, and $R$ ) phases in $\mathrm{NaNbO}_{3}$. The successive phase transitions from orthorhombic $\mathrm{Cmcm}\left(T_{1}\right)$ to other phases may be explained as follows. Comparison of neutron diffraction patterns of orthorhombic phases $\left(P, R, S\right.$, and $T_{2}$ phases $)$ reveals that $P, R$, and $S$ phases have additional superlattice reflections with respect to the orthorhombic $T_{2}(\mathrm{Cmcm})$ phase. We propose that these additional superlattice reflections arise due to condensation of the zone boundary phonons lying on the $T(M-R)$ line $(\mathbf{q}=1 / 2,1 / 2, g)$ as a function of temperature. The orthorhombic structure of $S, R$, and $P$ phases result from condensation of phonon modes $(\mathbf{q}=1 / 2,1 / 2, g)$ with $g=1 / 12$, $1 / 6$, and $1 / 4$, respectively. In other words, the structures of these various orthorhombic phases result from modulation of the high-symmetry cubic phase associated with phonon modes at $\mathbf{q}=(1 / 2,1 / 2, g)$. Further, freezing of all the $R_{25}$ modes along with a zone center phonon stabilizes the low-temperature ferroelectric rhombohedral phase. 
The continuous variation of lattice parameters with temperature for the orthorhombic $T_{1}, S$, and $R$ phases suggests that the successive phase transitions are of second order in nature. However, the phase transition from the orthorhombic $R$ to $P$ phase is of first order in nature. We recall that the space groups for orthorhombic $T_{1}$ and antiferroelectric $P$ phases are $C m \mathrm{~cm}$ and $\mathrm{Pbcm}$, respectively. Since the $\mathrm{Cmcm}$ to $\mathrm{Pbnm}$ transition follows the group-subgroup relation while $\mathrm{Pbnm}$ to $\mathrm{Pbcm}$ does not, we propose that the space group for both the orthorhombic $S$ and $R$ phases is $P b n m$ but with different unit cell modulation which accounts for all the superlattice reflections observed in neutron diffraction patterns.

The nature of the antiferrodistortive phase transition in materials exhibiting perovskite structure has attracted enormous attention $^{26-28}$ in the literature. Mainly, these phase transitions are driven by $R$ point instabilities. To the best of our knowledge, no attempt has been made to investigate the nature of the antiferrodistortive phase transition driven by $M$ point instabilities. With this aim, we have determined the effective exponent using the intensity of the superlattice reflection and tilt angle for sodium niobate of the tetragonal to cubic phase transition. The general relation between the order parameter $(p)$ and the intensity $(I)$ of these superlattice reflections is $I \propto p^{2}$. Figure 7 depicts the temperature dependence of the integrated intensity of the (310) superlattice reflection (associated with $M$ point instabilities) and also the tilt angle $(\alpha)$ as a function of temperature in the tetragonal phase. The data are fitted to the power laws $I \propto\left(T_{c}-T\right)^{2 \beta}$ and $\alpha \propto\left(T_{c}-T\right)^{\beta}$, which yields a critical exponent of $\beta \approx 0.32 \pm 0.03$ and transition temperature $\left(T_{c}\right)=953 \mathrm{~K}$. This behavior as seen in $\mathrm{SrTiO}_{3}$, $\mathrm{CaTiO}_{3}, \mathrm{Sr}_{x} \mathrm{Ca}_{1-x} \mathrm{TiO}_{3}$, and certain other perovskites ${ }^{26-28}$ is typical of tricritical phase transitions. The critical exponent $(\beta)$ for the cubic to tetragonal phase transition, as obtained from the temperature variation of the primary order parameter, is found to have a value of $1 / 3$. Thus, it is argued that this exponent is also a nearly tricritical transition.

Before we close, we would like to mention that by combining our present neutron diffraction data and earlier work ${ }^{7}$, we have modified the phase diagram proposed by pervious workers ${ }^{2}$ which was mainly based on x-ray diffraction studies. We now proceed to discuss the different features of our phase diagram given in Fig. 8. At high temperatures, the structure of $\mathrm{NaNbO}_{3}$ is cubic. First-principles calculations predicted the coexistence of zone center and zone boundary phonon instabilities in the cubic phase. On lowering the temperature, it undergoes successively transitions to tetragonal $\left(T_{2}\right.$ phase $)$ and orthorhombic ( $T_{1}$ phase) phases as a result of the condensation of zone boundary $M$ and $R$ point instabilities, respectively. Further, on lowering the temperature, the appearance of additional superlattice reflections in orthorhombic $S, R$, and $P$ phases with respect to the orthorhombic $\left(T_{1}\right)$ phase is linked to freezing of zone boundary phonons along the line $T$ (lying between the $M$ and $R$ points) with $\mathbf{q}=(1 / 2,1 / 2, g)$ with $g=1 / 12,1 / 6$, and $1 / 4$, respectively. The inelastic neutron scattering and $\mathrm{x}$-ray diffuse scattering experiments also suggest phonon instabilities along the $T$ line. Finally, $\mathrm{NaNbO}_{3}$ undergoes a rhombohedral phase transition due to condensation of both zone center and zone boundary $\left(R_{25}\right)$ modes. It is also important to state that for the orthorhombic $S$ and $R$ phases, we have also explored the possibilities for

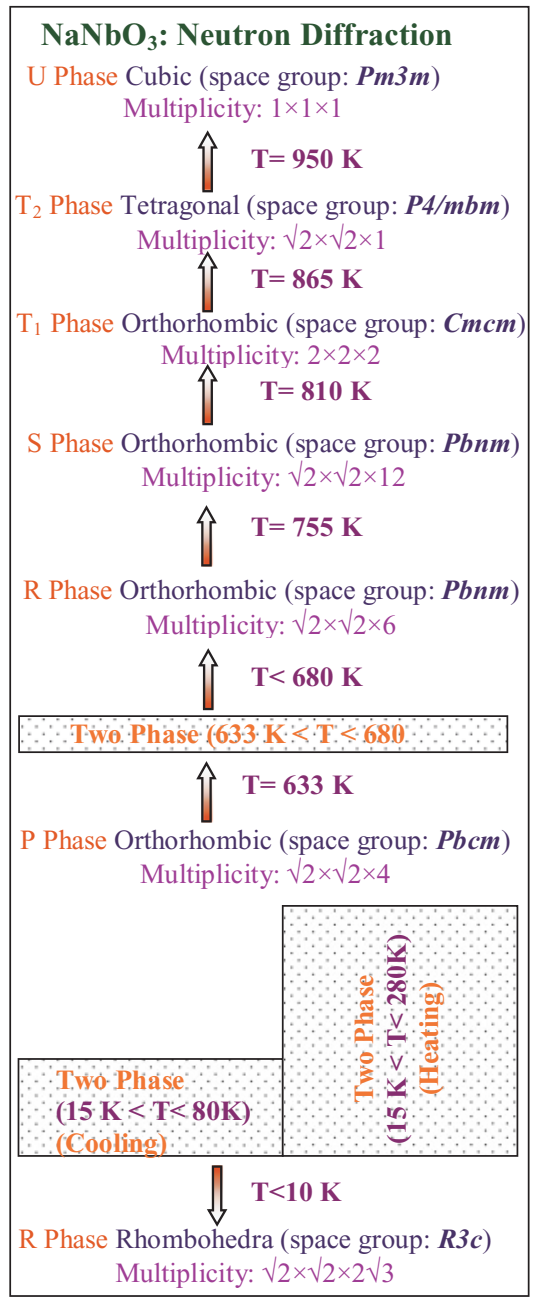

FIG. 8. (Color online) The phase diagram of $\mathrm{NaNbO}_{3}$ as a function of temperature after neutron diffraction studies. The structure and space group of $S$ and $R$ phases correspond to our own present work; while the existence of two phases below room temperature correspond to previous work.

the incommensurate-commensurate modulation using JANA software ${ }^{29}$ as observed for solid solutions of $\mathrm{Ba}$ or other perovskites. $^{30,31}$ We refined the diffraction data of the $S$ and $R$ phases with incommensurate modulated structures using JANA software; we find that the refined modulation vector does not vary significantly with temperature and the refined structure in both cases is quite close to the corresponding commensurate structure we reported above. Therefore, we do not consider the structures of the $S$ and $R$ phases as incommensurate, which is also consistent with the transmission electron study by Chen et $a l .{ }^{13}$ Single-crystal diffraction data are highly desirable for further confirmation.

\section{CONCLUSIONS}

In summary, we have established the structures and space groups of the technologically important sodium niobate up to $1075 \mathrm{~K}$. Distinct changes or vanishing of the intensity of superlattice reflections, as observed in the temperature dependence of neutron diffraction patterns, clearly revealed that $\mathrm{NaNbO}_{3}$ undergoes a series of phase transitions as a 
function of temperature, ranging from nonpolar antiferrodistortive to ferroelectric and antiferroelectric in nature. These phase transitions are mainly driven by zone boundary phonon instabilities at the $R$ and $M$ points and along the line $T$ (connecting the $M$ and $R$ points). Additional superlattice reflections are found to appear at $680 \mathrm{~K}$ ( $R$ phase) and $770 \mathrm{~K}$ ( $S$ phase), which could be indexed by using an intermediate long-period modulated orthorhombic structure whose lattice parameter is three and six times the lattice parameter of the $\mathrm{CaTiO}_{3}$ type Pbnm structure along $\langle 001\rangle$ directions. The correlation of superlattice reflections with the instability of zone boundary phonons is discussed. The discontinuous jump in lattice parameters at about $680 \mathrm{~K}$ and thermal hysteresis in the dielectric anomaly observed by Shirane et al. ${ }^{25}$ suggest that the transition from the orthorhombic $P$ to $R$ phase is of first order in nature, while other successive phase transitions are of second order. The phase diagram of $\mathrm{NaNbO}_{3}$ has been reviewed based on our detailed temperature-dependent neutron diffraction studies that resolved certain ambiguities in the literature.
${ }^{1}$ M. E. Lines and A. M. Glass, Principles and Application of Ferroelectrics and Related Materials (Clarendon, Oxford, 1977); Xu. Yuhuan, Ferroelectric Materials and Their Applications (NorthHolland/Elsevier Science, Amsterdam, 1991); L. G. Tejuca and J. L. G. Fierro, Properties and Applications of Perovskite-Type Oxides (Dekker, New York, 1993).

${ }^{2}$ E. Bousquet, M. Dawber, N. Stucki, C. Lichtensteiger, P. Hermet, S. Gariglio, J. M. Triscone, and P. Ghosez, Nature (London) 452, 723 (2008); T. Choi, Y. Horibe, H. T. Yi, Y. J. Choi, Weida Wu, and S.-W. Cheong, Nature Mater. 9, 253 (2010).

${ }^{3}$ Y. Saito, H. Takao, T. Tani, T. Nonoyama, K. Takatori, T. Homma, T. Nagaya, and M. Nakamura, Nature (London) 423, 84 (2004).

${ }^{4}$ E. Cross, Nature (London) 432, 24 (2004).

${ }^{5}$ E Valdez, C. B. de Araujo, and A. A. Lipovskii, Appl. Phys. Lett. 89, 031901 (2006); E. Hollenstein, M. Davis, D. Damjanovic, and Nava Setter, ibid. 87, 182905 (2006); E. L. Falcão-Filho, C. A. C. Bosco, G. S. Maciel, L. H. Acioli, Cid B. de Araújo, A. A. Lipovskii, and D. K. Tagantsev, Phys. Rev. B 69, 134204 (2004); G. S. Maciel, N. Rakov, Cid B. de Araujo, A. A. Lipovskii, and D. K. Tagantsev, Appl. Phys. Lett. 79, 5584 (2001).

${ }^{6}$ Yu. I. Yuzyuk, P. Simon, E. Gagarina, L. Hennet, D. Thiaudiere, V. I. Torgashev, S. I. Raevskya, I. P. Raevskii, L. A. Reznitchenko, and J. L. Sauvajol, J. Phys.: Condens. Matter 17, 4977 (2005); Yu. I. Yuyuk, E. Gagarina, P. Simon, L. A. Reznitchenko, L. Hennet, and D. Thiaudiere, Phys. Rev B 69, 144105 (2004); S Tripathi, D. Pandey, S K Mishra, and P. S. R. Krishna, ibid. 77, 052104 (2008); A. V. Ulinzheev, A. V. Leiderman, V. G. Smotrakov, V. Yu. Topolov, and O. E. Fesenko, Phys. Solid State 39, 972 (1997).

${ }^{7}$ S. K. Mishra, N. Choudhury, S. L. Chaplot, P. S. R. Krishna, and R. Mittal, Phys. Rev B 76, 024110 (2007), and reference therein.

${ }^{8}$ Y. Shiratori, A. Magrez, J. Dornseiffer, F. H. Haegel, C. Pithan, and R. Waser, J. Phys. Chem. B 43, 20122 (2005); Y. Shiratori, A. Magrez, W. Fischer, C. Pithan, and R. Waser, ibid. 111, 18493 (2007); Y. Shiratori, A. Magrez, M. Kato, K. Kasezawa, C. Pithan, and R. Waser, ibid. 112, 9610 (2008).

${ }^{9}$ C. N. W. Darlington and K. S. Knight, Physica B 266, 368 (1999); Acta Crystallogr. Sect. B 55, 24 (1999); C. N. W. Darlington and H. D. Megaw, ibid. 29, 2171 (1973); C. N. W. Darlington, Solid State Commun. 29, 307 (1979); L. E. Cross and B. J. Nicholson, Philos. Mag. 46, 212 (1955); A. C. Sakowski-Cowley, Ph.D. Thesis, University of Cambridge, 1967.

${ }^{10}$ M. Ahtee, A M. Glazer, and H. D Megaw, Philos. Mag. 26995 (1972); A. M. Glazer and H. D. Megaw, ibid. 251119 (1972); Acta Crystallogr. Sect. A. 29, 489 (1973); A. C. Sakowski-Cowely,
K. Lukazewicz, and H. D. Megaw, Acta Crystallogr. Sect. B 25, 851 (1969);

${ }^{11}$ I. Lefkowitzk, K. Lukazewicz, and H. D. Megaw, Acta. Crystllogr. 20, 670 (1966); F. Denoyer, M. Lambert, and R. Comes, Solid State Commun. 18, 441 (1976), and references therein.

${ }^{12}$ I. Tomeno, Y. Tsunoda, K. Oka, M. Matsuura, and M. Nishi, Phys. Rev. B 80104101 (2009); K. Ishida and G. Honj, J. Phys. Soc. Jpn. 34, 1279 (1973).

${ }^{13}$ J. Chen and D. Feng, Phys. Status Solidi A 109, 171 (1988); 109, 427 (1988).

${ }^{14}$ W. Zhong and D. Vanderbilt, Phys. Rev. Lett. 74, 2587 (1995).

${ }^{15}$ O. Diéguez, K. M. Rabe, and D. Vanderbilt, Phys. Rev. B 72, 144101 (2005).

${ }^{16}$ D. Vanderbilt and W. Zhong, Ferroelectrics 181, 206 (1998).

${ }^{17}$ W. Zhong, R. D. King-Smith, and D. Vanderbilt, Phys. Rev. Lett. 72, 3618 (1994).

${ }^{18}$ R. D. King-Smith and D. Vanderbilt, Phys. Rev. B 49, 5828 (1994).

${ }^{19}$ Throughout this paper, we use the nomenclature of the specific points and lines in the Brillouin zone of the parent cubic perovskite phase (space group $p m \overline{3} \mathrm{~m}$ ) only. The term "superlattice reflection" is used with respect to the parent cubic phase.

${ }^{20}$ A. M. Glazer, Acta Crystallogr., Sect. B 28, 3384 (1972); Acta Crystallogr. 31, 756 (1975).

${ }^{21}$ P. Fischer et al., Physica B 276-278, 146 (2000); [http://sinq.web.psi.ch/hrpt]

${ }^{22}$ J. Rodriguez-Carvajal Physica B 192, 55 (1993).

${ }^{23}$ J. Hutton, R. J. Nelms, G. M. Meyer, and V. R. Eiriksson, J. Phys. C 12, 5393 (1979).

${ }^{24}$ H. T. Stokes, D. M. Hatch, and B. J. Campbell, computer code ISOTROPY (2007) [http://stokes.byu.edu/isotropy.html].

${ }^{25}$ G. Shirane, R. Newnham, and R. Pepinsky, Phys. Rev. 96, 581 (1954).

${ }^{26}$ A. D. Bruce and R. A. Cowely, Adv. Phys. 29, 219 (1980).

${ }^{27}$ S. A. T. Redfern, J. Phys.: Condens. Matter 8, 8267 (1996); B. J. Kennedy, C. J. Howard, and B. C. Chakoumakos, ibid. 11, 1479 (1999).

${ }^{28}$ S K Mishra and D Pandey, Phys. Rev. B 79, 174111 (2009).

${ }^{29}$ V Petříček, M. Dušek , and L Palatinus, computer code JANA2000 (Institute of Physics, Praha, Czech Republic).

${ }^{30}$ G. Baldinozziy, D. Grebillez, Ph. Sciauy, J.-M. Kiatyx, J. Moretk, and J-F Berar, J. Phys.: Condens. Matter 10, 6461 (1998); J. M. Kia, G. Calvarin, and J. Schneck, Phys. Rev. B 49, 776 (1994).

${ }^{31}$ H. Z. Cummins, Phys. Rep. 185, 211 (1990). 